\title{
Introduction to Session 12: Advances in Enzyme Science and Technology
}

\author{
K. Thomas Klasson
}

Published online: 27 March 2009

(C) Humana Press 2009

As this meeting has grown, several of the most popular topics have been spilt into two sessions in order so that more presenters could give talks and to allow the audience to hear more research angles from a variety of sources. These two sessions focused on the enzymatic deconstruction of biomass and how it works synergistically with pretreatment to promote sugar release for bioconversion. The sessions presented the multi-front efforts that are underway to improve economic production and discover new enzymes with the eventual goal of developing a biological process for complete biomass deconstruction. The first session was held on Monday and the second part of the session was held on Wednesday. Session 12 was chaired by David Wu of Rochester University and Colin Mitchinson of Genencor.

Nazmul Karim, representing a research group from Texas Tech, gave a presentation on the "Screening for New Cellulase Enzymes to Enhance the Synergistic Effect by Codisplaying Cellulases on the Surface of E. coli, LY01." The presenter gave a talk that described the use of a cDNA library from Clostridium cellulyticum expressed on the surface of Escherichia coli strain LY01. This method was employed to screen for new cellulases, which showed enhanced enzymatic synergistic effect. The surface-displayed enzymes were quantitatively measured using flow cytometric analysis.

The research group from Novozymes North America, represented by Frank Haagensen, presented a talk titled "Performance Data of Novozymes New Cellulase Systems." The presentation highlighted the fact that the company had taken up the US Department of Energy's 2001 challenge to lower the enzyme cost of cellulose hydrolysis. They were able to reduce the cost 30 -fold. The new enzymes were not only cheaper but also showed that the performance was improved if certain process conditions (e.g., $\mathrm{pH}$ and temperature) were optimized.

David Wilson from Cornell University discussed how the five structures of the catalytic domain had been determined in a presentation titled "Family GH-6 Cellulases Do Not Seem to Possess a Catalytic Base." Wilson said that his group's work with site-directed mutagenesis of a specific residue and azide rescue experiments had shown that the acid

K. T. Klasson $(\bowtie)$

USDA, Agricultural Research Service, New Orleans, LA, USA

e-mail: Thomas.Klasson@ARS.USDA.GOV 
residues (which act as catalytic base) in the active sites from Trichoderma reesei and Trichoderma fusca may not come from a single common residue but may be shared among several residues in the Trichoderma family.

The cellulase enzyme system in "Clostridium thermocellum" has more than 70 subunits. The researchers from University of Rochester, represented by David Wu, gave a presentation titled "The Clostridium thermocellum Cellulosome, a Molecular Machine for Cellulose Degradation." Wu described that the bacterium may modulate the biosynthesis of its enzyme components to optimize its activity on an available biomass substrate. This group's work indicated that regulation of the degradative enzymes can be accomplished through soluble sugars generated from the insoluble substrate by the action of the enzymes.

Babu Raman of Oak Ridge National Laboratory presented his work on "Biomass Ethanol from Clostridium thermocellum-A Systems Biology Analysis." Just as in the previous talk, his work focused on the bacterium $C$. thermocellum. Raman had used microarray technology to probe the genetic expression of $C$. thermocellum during cellulose and cellobiose anaerobic fermentation. Broadly, genes involved in energy production, translation, glycolysis and amino acid, nucleotide, and coenzyme metabolism displayed a progressively decreasing trend in gene expression. In comparison, genes involved in cell structure and motility, chemotaxis, signal transduction, transcription, and cellulosomal genes showed an increasing trend in gene expression.

The final presentation was given by Aaron Kelley of Genencor and was titled "Design and Development of an Enzyme Products for Cellulosic Biomass Conversion." Kelley discussed the steps of launching a commercial product to the cellulosic biomass market and his group's experience taking the necessary tests. The talk included the design of an enzyme system, performance testing, and validation, confirming reproducible production quality and delivering on needed production cost reductions. 